\title{
THE K-THEORY OF HEEGAARD-TYPE QUANTUM 3-SPHERES
}

\author{
Dedicated to the memory of Olaf Richter.
}

\author{
PaUl Baum \\ Mathematics Department, McAllister Building \\ The Pennsylvania State University \\ University Park, PA 16802, USA \\ e-mail: baum@math.psu.edu
}

\section{Piotr M. Hajac}

Instytut Matematyczny, Polska Akademia Nauk

ul. Śniadeckich 8, Warszawa, 00-956 Poland

and

Katedra Metod Matematycznych Fizyki, Uniwersytet Warszawski

ul. Hoża 74, Warszawa, 00-682 Poland

http://www.fuw.edu.pl/ pmh

\section{RAINER MATTHES}

Katedra Metod Matematycznych Fizyki, Uniwersytet Warszawski ul. Hoża 74, Warszawa, 00-682 Poland

e-mail: matthes@itp.uni-leipzig.de

\author{
WOJCIECH SZYMAŃSKI \\ School of Mathematical and Physical Sciences, University of Newcastle \\ Callaghan, NSW 2308, Australia \\ e-mail: wojciech@frey.newcastle.edu.au
}

\begin{abstract}
We use a Heegaard splitting of the topological 3-sphere as a guiding principle to construct a family of its noncommutative deformations. The main technical point is an identification of the universal $C^{*}$-algebras defining our quantum 3-spheres with an appropriate fiber product of crossed-product $C^{*}$-algebras. Then we employ this result to show that the $K$-groups of our family of noncommutative 3 -spheres coincide with their classical counterparts.
\end{abstract}




\section{Introduction}

It seems we are in a period of development of Noncommutative Geometry when new constructions of quantum spheres appear in abundance. Perhaps a key reason for this richness of examples is that a wish to preserve some particular additional structures that live on a topological space often leads to, or allows for, significantly different types of noncommutative deformations. The flexibility and scope of the language of noncommutative $C^{*}$-algebras leaves enough room to reflect much more in the structure of a $C^{*}$-algebra than just pure topology of the underlying space.

For instance, the concept of the $S U(2)$ group structure on the 3-sphere uniquely determined (under some natural assumptions) its deformation into the quantum group $S U_{q}(2)$ [W-SL87]. Among recent examples, we have the classification of quantum deformations of $S^{3}$ that preserve certain classical cohomological conditions [CD02, CD03]. Both new and earlier examples of noncommutative spheres are beautifully surveyed in [D-L03], and we refer the reader for more details and references therein.

Our present work stems from the idea of the decomposition of $S^{3}$ into two solid tori that corresponds to the local triviality of the Hopf fibration $S^{3} \rightarrow S^{2}$. It is known as a Heegaard splitting of $S^{3}$. This decomposition principle was first explored in [M-K91] yielding the quantum-torus type deformation $S_{\theta}^{3}$, and, recently, in [CM02, HMS] producing the quantum-disc type deformation $S_{p q}^{3}$. Both approaches were unified into $S_{p q \theta}^{3}$ in [BHMS], where the coordinate algebra $\mathcal{O}\left(S_{p q \theta}^{3}\right)$ is studied from Hopf-Galois and index theory point of view. Herein, we compute the $K$-theory of the universal enveloping $C^{*}$-algebra of $\mathcal{O}\left(S_{p q \theta}^{3}\right)$, which is the main result of this paper.

To understand better the $C^{*}$-algebraic structure we work with, note first that the aforementioned Heegaard splitting of $S^{3}$ decomposes it into the glueing over the boundary torus of two copies of a solid torus which is the Cartesian product of a disc and circle. Our noncommutative 3 -sphere is an analogous glueing (represented by the fiber product of algebras) of two copies of a quantum solid torus. We represent a quantum solid torus by the crossed product of the Toeplitz algebra $\mathcal{T}$ by a natural action of $\mathbb{Z}$ determined by the parameter $\theta$, i.e., $\mathcal{T} \rtimes_{\theta} \mathbb{Z}$. Just as the standard epimorphism $\mathcal{T} \rightarrow C\left(S^{1}\right)$ allows one to think of $S^{1}$ as the boundary of a quantum disc, the natural epimorphism $\mathcal{T} \rtimes_{\theta} \mathbb{Z} \rightarrow C\left(S^{1}\right) \rtimes_{\theta} \mathbb{Z}$ permits one to view the noncommutative torus as the boundary of a quantum solid torus. This is the way the Toeplitz and irrational rotation $C^{*}$-algebras are used to assemble a new $C^{*}$-algebra along the lines of the topological idea of a Heegaard splitting of $S^{3}$.

Before embarking on a computation of the $K$-groups of noncommutative deformations of topological spaces, it is reasonable to ask under which general conditions we have a guarantee that the $K$-groups of the deformed $C^{*}$-algebras remain the same as the $K$-groups of the initial $C^{*}$-algebras. An example of such general conditions was provided in [R-MA93]. On the other hand, even a very standard deformation of a torus into a quantum torus $T_{\theta}^{2}$, after passing to the quotient by the antipodal action $T_{\theta}^{2} / Z_{2}$, leads to different $K$-theory [BEEK91, BEEK92, BK92]:

$$
K_{0}\left(C\left(T_{\theta}^{2} / Z_{2}\right)\right)=K_{0}\left(\left(C\left(S^{1}\right) \rtimes_{\theta} \mathbb{Z}\right)^{Z_{2}}\right) \cong \mathbb{Z}^{6} \neq \mathbb{Z}^{2} \cong K^{0}\left(S^{2}\right)=K_{0}\left(C\left(T^{2} / Z_{2}\right)\right) .
$$

Therefore, it is in general not true that the $K$-theory remains unchanged with deformations. 
As it seems unlikely that our example fits into Rieffel's framework [R-MA93], we prove directly that the $K$-groups of our family of quantum spheres coincide with their classical counterparts.

The overall mathematical structure of this article is much in line with [M-K91]. Sectionwise, it is organized as follows. We begin with preliminaries where notation is fixed and basic facts recalled. Also in preliminaries, we warm up for the $K$-theory computation by analysing the classical geometric roots of the Mayer-Vietoris six-term exact sequence in the $K$-theory of $C^{*}$-algebras. Then we proceed with the main technical contents of the paper, which is the determination of key $C^{*}$-algebraic features of our quantum spheres. First we show that the universal $C^{*}$-algebra remains unchanged if we set two of our parameters to zero, i.e., $C\left(S_{p q \theta}^{3}\right) \cong$ $C\left(S_{00 \theta}^{3}\right)$. Next we prove the latter $C^{*}$-algebra to be isomorphic with a fiber product (pullback) of two crossed products. These two steps make it possible to compute the $K$-groups of $C\left(S_{p q \theta}^{3}\right)$ by standard methods of the $K$-theory of operator algebras [W-NE93, B-B98].

\section{Preliminaries}

Throughout the paper we use the jargon of noncommutative geometry referring to quantum spaces as objects dual to noncommutative algebras in the sense of the Gelfand-Naimark correspondence between spaces and function algebras. The algebras are assumed to be associative and over $\mathbb{C}$. The notation $C_{0}$ (locally compact Hausdorff space) means the algebra of vanishingat-infinity continuous functions on this space. This algebra is non-unital unless the space is compact, in which case we skip the zero subscript. (In the noncommutative setting, the unitality of a $C^{*}$-algebra is viewed as the compactness of a quantum space.) By $\mathcal{O}$ (quantum space) we denote the polynomial unital *-algebra of a quantum space defined by generators and relations, and by $C$ (quantum space) the corresponding $C^{*}$-algebra.

The latter means the universal enveloping $C^{*}$-algebra (or the $C^{*}$-completion, or the $C^{*}$ closure) of a $*$-algebra in the following sense. Let $\mathcal{O}$ be a unital $*$-algebra such that the set $\operatorname{Rep}(\mathcal{O})$ of all its bounded $*$-representations is non-empty, and such that

$$
\forall x \in \mathcal{O}: \sup \{\|\varrho(x)\| \mid \varrho \in \operatorname{Rep}(\mathcal{O})\}=:\|x\|_{\text {sup }}<\infty .
$$

Then \|\|$_{\text {sup }}$ defines a norm on the quotient algebra $\mathcal{O} /\left\{x \in \mathcal{O} \mid\|x\|_{\text {sup }}=0\right\}$ whose norm completion is the aforementioned universal enveloping $C^{*}$-algebra.

The thus constructed $C^{*}$-algebras indeed enjoy a very important universality property which we use a number of times in this paper. One can show that, if $C$ is the universal enveloping $C^{*}$-algebra of $\mathcal{O}$ and $A$ is a $C^{*}$-algebra, then for any $*$-homomorphism $\mathcal{O} \rightarrow A$ there exists a unique $C^{*}$-homomorphism $C \rightarrow A$ such that the diagram

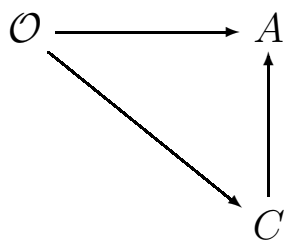

is commutative. (Here the diagonal map is the canonical quotient map combined with the injection of the quotient algebra into its norm completion.) This universality property is very 
useful because it allows one to define $C^{*}$-homomorphisms simply by appropriately specifying them on generators.

On the other hand, for $K$-theoretical computations it is useful to present a $C^{*}$-algebra as a fiber-product or crossed-product $C^{*}$-algebra. We denote the fiber product of algebras by a decorated direct sum, and the crossed product of an algebra $A$ by a group $G$ as $A \rtimes G$. For generalities on the fiber products (pullbacks) of $C^{*}$-algebras we recommend [P-GK99], and for $C^{*}$-algebraic crossed products we refer to [B-B98, Chapter V] and [P-GK79, Chapter 7]. Thanks to Theorem 3.2, our calculation of $K$-groups rests on the standard tools for the $K$-theory of fiber products and crossed products.

\section{$1.1 \quad$ A Heegaard splitting of $S^{3}$}

Splitting and glueing topological spaces along 2-spheres or 2-tori are standard procedures in the study of 3-dimensional manifolds. In the case of $S^{3}$, we have the well-known Heegaard splittings. They present $S^{3}$ as two copies of a solid torus glued along their boundaries.

More precisely, the situation is as follows (see [N-GL97, Section 0.3] and [HMS] for related details). Define

$$
X=\left\{\left(z_{1}, z_{2}\right) \in \mathbb{C}^{2}\left|\left(1-\left|z_{1}\right|^{2}\right)\left(1-\left|z_{2}\right|^{2}\right)=0,\right| z_{i} \mid \leq 1\right\} .
$$

It is clear that $X$ is a glueing of two solid tori along their boundaries. On the other hand, $S^{3}=\left\{\left.\left(c_{1}, c_{2}\right) \in \mathbb{C}^{2}|| c_{1}\right|^{2}+\left|c_{2}\right|^{2}=1\right\}$ is the usual round 3-sphere. To see that they are indeed homeomorphic, note that the following formulas give continuous and mutually inverse maps between these two spaces:

$$
\begin{gathered}
f\left(\left(z_{1}, z_{2}\right)\right)=\left(\left|z_{1}\right|^{2}+\left|z_{2}\right|^{2}\right)^{-\frac{1}{2}}\left(z_{1}, z_{2}\right), \\
g\left(\left(c_{1}, c_{2}\right)\right)=\frac{\sqrt{2}\left(c_{1}, c_{2}\right)}{\sqrt{1+\left.|| c_{1}\right|^{2}-\left|c_{2}\right|^{2} \mid}} .
\end{gathered}
$$

It is a basic corollary of Bott periodicity [B-PF72] that for the $n$-sphere $S^{n}$, the Chern character

$$
c h: K^{*}\left(S^{n}\right) \longrightarrow H^{*}\left(S^{n} ; \mathbb{Q}\right)
$$

maps $K^{*}\left(S^{n}\right)$ isomorphically onto $H^{*}\left(S^{n} ; \mathbb{Z}\right) \subseteq H^{*}\left(S^{n} ; \mathbb{Q}\right)$. In particular,

$$
K^{j}\left(S^{n}\right)=\left\{\begin{array}{cc}
\mathbb{Z} \oplus \mathbb{Z} & j=0 \\
0 & j=1
\end{array}, \quad \text { for } n \text { even, and } \quad K^{j}\left(S^{n}\right)=\left\{\begin{array}{cc}
\mathbb{Z} & j=0 \\
\mathbb{Z} & j=1
\end{array} \text { for } n\right. \text { odd. }\right.
$$

In the special case of $S^{3}$, any complex vector bundle on $S^{3}$ is trivial. (This is because any bundle over $S^{3}$ can be clutched over $S^{2}$ from trivial bundles on 3-dimensional balls and the second homotopy group of any compact connected Lie group is zero.) Thus $K^{0}\left(S^{3}\right)=\mathbb{Z}$ and a generator is the trivial line bundle $S^{3} \times \mathbb{C}$. For $K^{1}\left(S^{3}\right)$, observe that, by Bott periodicity, $\pi_{3}(U(n))=\mathbb{Z}$ for $n \geq 2$. Hence $K^{1}\left(S^{3}\right)=\mathbb{Z}$ with a generator given by the map

$$
\left(z_{1}, z_{2}\right) \longmapsto\left(\begin{array}{cc}
z_{1} & -\bar{z}_{2} \\
z_{2} & \bar{z}_{1}
\end{array}\right) \text {. }
$$


From the $C^{*}$-algebra point of view, $K_{0}\left(C\left(S^{3}\right)\right)$ is generated by the unit of $C\left(S^{3}\right)$ and $K_{1}\left(C\left(S^{3}\right)\right)$ is generated by the 2-by-2 unitary matrix given above, now with $z_{1}, z_{2}$, viewed as the coordinate functions on $S^{3}$. If we view $S^{3}$ as the union of two solid tori, glued along their boundaries, then there is the 6-term Mayer-Vietoris exact sequence

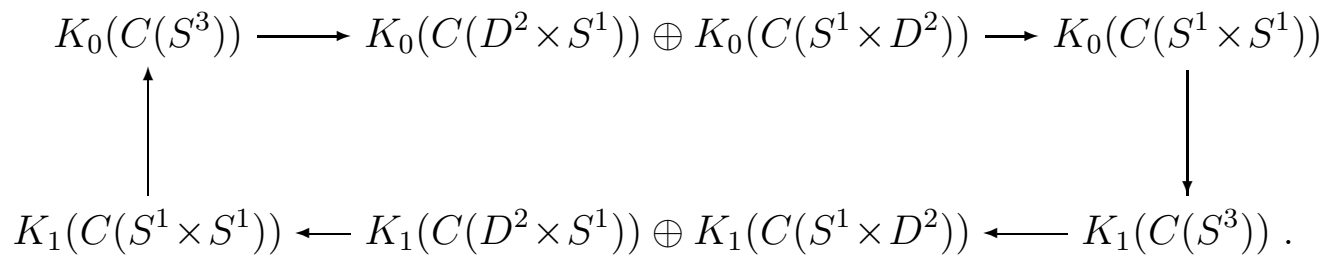

The $K$-groups of $S^{3}$ could be calculated from this diagram. In the noncommutative case, this is how we shall calculate the $K$-groups for our examples of quantum 3 -spheres.

\subsection{The Toeplitz and irrational rotation $C^{*}$-algebras}

The Toeplitz and irrational rotation $C^{*}$-algebras $\mathcal{T}$ and $C\left(S^{1}\right) \rtimes_{\theta} \mathbb{Z}$ are among the best studied examples of operator algebras. They admit natural geometrical interpretations as a quantum disc and a quantum torus, respectively. As explained in the introduction, they become basic building blocks in our construction of a quantum 3-sphere just as a disc and torus can be used to build up $S^{3}$. Herein, we recall some basic facts about these algebras.

Let $\mathcal{O}\left(D_{q}\right)$ stand for the unital $*$-algebra generated by $z, z^{*}$ satisfying

$$
z^{*} z-q z z^{*}=1-q, \quad 0 \leq q<1
$$

Denote by $\left\{e_{k}\right\}_{k \in \mathbb{N}}$ an orthonormal basis of a Hilbert space. Up to the unitary equivalence, $\mathcal{O}\left(D_{q}\right)$ has the following irreducible $*$-representations in bounded operators [KL93]:

$$
\pi(z) e_{k}=\sqrt{1-q^{k+1}} e_{k+1}, k \in \mathbb{N}, \quad \pi_{\lambda}(z)=\lambda, \lambda \in U(1) .
$$

Using this classification, one can show that the extension of the representation $\pi$ to the universal enveloping $C^{*}$-algebra $C\left(D_{q}\right)$ of $\mathcal{O}\left(D_{q}\right)$ is faithful [KL93, p.14]. A straightforward argument proves that the norm of $z$ in $C\left(D_{q}\right)$ is 1 . (The same argument is used to prove (2.15).) For $q=1$ this norm condition is no longer a consequence of the commutation relation, but can be naturally assumed to hold, as it always holds automatically for $0 \leq q<1$. The norm condition combined with the commutativity of $z$ and $z^{*}$ yields the $C^{*}$-algebra of continuous functions on the unit disc $D$. This is a motivation for calling $C\left(D_{q}\right)$ the $C^{*}$-algebra of a quantum disc [KL93].

On the other hand, $C\left(D_{0}\right)$ is, by construction, the well-known Toeplitz algebra $\mathcal{T}$. The faithful representation $\pi$ allows us to view $\mathcal{T}$ as the operator algebra generated by the unilateral shift. It appears quite interesting that, for $0<q<1$, we can always re-scale the generator $z$ by dividing it (on the right) by its absolute value to obtain the usual generator of $\mathcal{T}$ that we have for $q=0$. This is precisely the phenomenon that we use to prove Theorem 2.8 (see (2.46)).

Next, let $\mathcal{O}\left(T_{\theta}^{2}\right)$ denote the unital $*$-algebra generated by $x$ and $y$ satisfying

$$
x x^{*}=1=x^{*} x, \quad y y^{*}=1=y^{*} y, \quad x y=e^{2 \pi i \theta} y x, \quad \theta \in[0,1[.
$$


The universal enveloping $C^{*}$-algebra $C\left(T_{\theta}^{2}\right)$ of $\mathcal{O}\left(T_{\theta}^{2}\right)$ coincides with the crossed-product algebra $C\left(S^{1}\right) \rtimes_{\theta} \mathbb{Z}$. For $\theta=0$ we get the algebra of continuous functions on the 2 -torus $T^{2}$. Hence, for $\theta \neq 0$, the quantum space $T_{\theta}^{2}$ is called a noncommutative torus. The situation is particularly interesting when $\theta$ is irrational. Therefore, the irrationality of $\theta$ is often automatically assumed, in which case the crossed product $C\left(S^{1}\right) \rtimes_{\theta} \mathbb{Z}$ goes under the name irrational rotation $C^{*}$-algebra [R-MA90].

\subsection{The Mayer-Vietoris sequence for the K-theory of $\mathrm{C}^{*}$-algebras}

Let

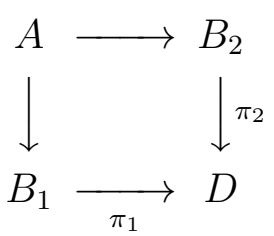

be a commutative diagram of $C^{*}$-algebras in which each homomorphism is surjective and $A:=$ $\left\{\left(b_{1}, b_{2}\right) \in B_{1} \oplus B_{2} \mid \pi_{1}\left(b_{1}\right)=\pi_{2}\left(b_{2}\right)\right\}$. Then $A$ is again a $C^{*}$-algebra and it is called the fiber product (or pullback) of $B_{1}$ and $B_{2}$ over $D$. In case the $C^{*}$-algebras $B_{1}$ and $B_{2}$ are commutative, an argument of Atiyah and Hirzebruch [AH62, p.32], allows one to construct the six-term $K$-theory exact sequence

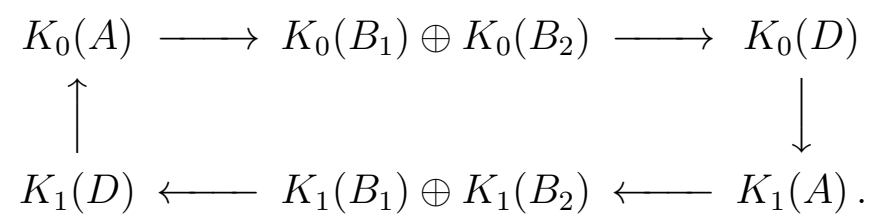

We make a very slight change in the Atiyah-Hirzebruch argument (so that no partition of unity is used) and then observe that their construction applies to the case when the $C^{*}$-algebras are not required to be commutative.

First, recall the classical construction. Here $X$ is a locally compact Hausdorff space and $X_{1}, X_{2}$, are closed subsets of $X$ with $X=X_{1} \cup X_{2}$. Let $\tilde{X}$ be the subset of $X \times[0,1]$ :

$$
\tilde{X}=X_{1} \times\{0\} \cup\left(X_{1} \cap X_{2}\right) \times[0,1] \cup X_{2} \times\{1\} .
$$

Thus $\tilde{X}$ is the disjoint union of $X_{1}$ and $X_{2}$ with a cylinder connecting the two copies of $X_{1} \cap X_{2}$

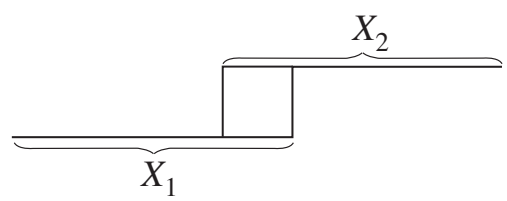

that is topologized as a subspace of $X \times[0,1]$. Consider now the six-term $K$-theory exact sequence $[\mathrm{K}-\mathrm{M} 78, \mathrm{p} .90]$ for $\left(\tilde{X}, X_{1} \times\{0\} \cup X_{2} \times\{1\}\right)$ :

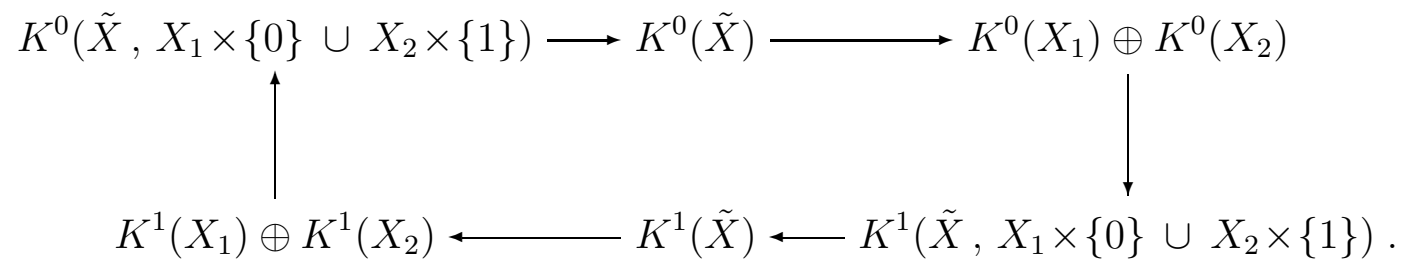


Next, let us use the notation $Y / Y^{\prime}$ to signify the space $Y$ with its subspace $Y^{\prime}$ shrunk to a point and observe that $\tilde{X} /\left(X_{1} \times\{0\} \cup X_{2} \times\{1\}\right)$ is the suspension of $X_{1} \cap X_{2}$. Hence we conclude that

$$
K^{j}\left(\tilde{X}, X_{1} \times\{0\} \cup X_{2} \times\{1\}\right)=K^{1-j}\left(X_{1} \cap X_{2}\right) .
$$

Furthermore, we can prove that the restriction of the projection $X \times[0,1] \rightarrow X$ to $\tilde{X} \rightarrow X$ gives an isomorphism in $K$-theory:

$$
K^{j}(X) \stackrel{\cong}{\longrightarrow} K^{j}(\tilde{X}), \quad j=0,1 .
$$

Indeed, the map $\tilde{X} \rightarrow X$ maps $X_{1} \times\{0\} \cup\left(X_{1} \cap X_{2}\right) \times[0,1]$ to $X_{1}$ and so gives a map of pairs

$$
\left(\tilde{X}, X_{1} \times\{0\} \cup\left(X_{1} \cap X_{2}\right) \times[0,1]\right) \rightarrow\left(X, X_{1}\right)
$$

Hence the six-term $K$-theory exact sequence for $\left(X, X_{1}\right)$ maps to the six-term $K$-theory exact sequence for $\left(\tilde{X}, X_{1} \times\{0\} \cup\left(X_{1} \cap X_{2}\right) \times[0,1]\right)$. Since $\tilde{X} /\left(X_{1} \times\{0\} \cup\left(X_{1} \cap X_{2}\right) \times[0,1]\right)=X / X_{1}$ and since $X_{1} \times\{0\} \cup\left(X_{1} \cap X_{2}\right) \times[0,1] \rightarrow X_{1}$ is clearly a homotopy equivalence, the Five Isomorphisms Lemma now applies to end the proof of (1.18). Combining this with (1.17) and the six-term exact sequence for $\left(\tilde{X}, X_{1} \times\{0\} \cup X_{2} \times\{1\}\right)$ now yields the desired Mayer-Vietoris sequence (1.14).

We shall now show that the reasoning used in the commutative case applies basically unchanged to the general case. Let $\tilde{A} \subseteq B_{1} \oplus B_{2} \oplus C([0,1], D)$ be

$$
\tilde{A}=\left\{\left(b_{1}, b_{2}, \omega\right) \mid b_{1} \in B_{1}, b_{2} \in B_{2}, \omega(0)=\pi_{1}\left(b_{1}\right), \omega(1)=\pi_{2}\left(b_{2}\right)\right\} .
$$

As usual, $C([0,1], D)$ is the $C^{*}$-algebra of all continuous maps from $[0,1]$ to $D$. Similarly, put

$$
C_{0}(] 0,1[, D)=\{\omega \in C([0,1], D) \mid \omega(0)=\omega(1)=0\} .
$$

Consider now the short exact sequence of $C^{*}$-algebras

$$
0 \longrightarrow C_{0}(] 0,1[, D) \longrightarrow \tilde{A} \longrightarrow B_{1} \oplus B_{2} \longrightarrow 0, \quad\left(b_{1}, b_{2}, \omega\right) \longmapsto\left(b_{1}, b_{2}\right)
$$

The six-term $K$-theory exact sequence of this short exact sequence of $C^{*}$-algebras plus the identifications

$$
K_{j}(C(] 0,1[, D))=K_{1-j}(D), \quad K_{j}(\tilde{A})=K_{j}(A), \quad j=0,1,
$$

then yield the desired Mayer-Vietoris exact sequence. The first of these identifications is immediate because $C(] 0,1[, D)$ is the suspension of $D$. For the second, we wish to prove that the evident map

$$
A \longrightarrow \tilde{A}, \quad\left(b_{1}, b_{2}\right) \longmapsto\left(b_{1}, b_{2}, \underline{\pi_{1}\left(b_{1}\right)}\right),
$$

where $\underline{\pi_{1} b_{1}}$ is the constant path at $\pi_{1}\left(b_{1}\right)=\pi_{2}\left(b_{2}\right)$, gives a $K$-theory isomorphism. Let $I_{1}$ be the kernel of

$$
A \longrightarrow B_{1}, \quad\left(b_{1}, b_{2}\right) \longmapsto b_{1}
$$

Consider the pair $\left(A, I_{1}\right)$, and note that $I_{1} \cong \operatorname{Ker}\left(\pi_{2}: B_{2} \rightarrow D\right)$. The image of $I_{1}$ in $\tilde{A}$ is $\left\{\left(0, b_{2}, \underline{0}\right) \mid \pi_{2}\left(b_{2}\right)=0\right\}$. (Here $\underline{0}$ denotes the constant path at $0 \in D$.) Thus the image of $I_{1}$ in 
$\tilde{A}$ is an ideal in $\tilde{A}$. By a slight abuse of notation, denote the image of $I_{1}$ in $\tilde{A}$ also by $I_{1}$. The map of pairs

$$
\left(A, I_{1}\right) \longrightarrow\left(\tilde{A}, I_{1}\right), \quad\left(b_{1}, b_{2}\right) \longmapsto\left(b_{1}, b_{2}, \underline{\pi_{1}\left(b_{1}\right)}\right),
$$

maps the six-term $K$-theory exact sequence [B-B98, p.67] for $\left(A, I_{1}\right)$ to the six-term $K$-theory exact sequence for $\left(\tilde{A}, I_{1}\right)$. Finally, let us compare $A / I_{1}=B_{1}$ and $\tilde{A} / I_{1}$. We have

$$
\begin{aligned}
\tilde{A} / I_{1} & =\left\{\left(b_{1}, b_{2}, \omega\right) \mid \omega(0)=\pi_{1}\left(b_{1}\right), \omega(1)=\pi_{2}\left(b_{2}\right)\right\} /\left\{\left(0, b_{2}, \underline{0}\right) \mid \pi_{2}\left(b_{2}\right)=0\right\} \\
& =\left\{\left(b_{1}, \omega\right) \mid b_{1} \in B_{1} \text { and } \omega:[0,1] \rightarrow D \text { has } \omega(0)=\pi_{1}\left(b_{1}\right)\right\} .
\end{aligned}
$$

It is now clear that the $C^{*}$-algebras $A / I_{1}=B_{1}$ and $\tilde{A} / I_{1}$ are homotopy equivalent. Therefore, an analogue of the argument proving (1.18) applies to prove the desired identification $K_{j}(\tilde{A}) \cong$ $K_{j}(A), j=0,1$. This completes the construction of the Mayer-Vietoris exact sequence for the $K$-theory of $C^{*}$-algebras.

\section{The universal $\mathrm{C}^{*}$-algebras}

In this section, we show the existence of the universal $C^{*}$-algebra for relations that we interpret as defining a 3-parameter family of noncommutative 3-spheres. By definition, the topology of the parameter space is that of a solid torus, but it turns out that identifying points in this space that give isomorphic $C^{*}$-algebras largely reduces its size. We begin by defining the polynomial *-algebras of our noncommutative 3 -spheres [BHMS].

Definition 2.1. The unital $*$-algebra $\mathcal{O}\left(S_{p q \theta}^{3}\right)$ is the quotient of the free *-algebra generated by elements $a$ and $b$ by the $*$-ideal generated by the following relations:

$$
\begin{array}{ll}
\left(1-a a^{*}\right)\left(1-b b^{*}\right)=0 & \text { the sphere equation, } \\
a^{*} a=p a a^{*}+1-p, \quad b^{*} b=q b b^{*}+1-q & \text { quantum-disc relations, } \\
a b=e^{2 \pi i \theta} b a, \quad a b^{*}=e^{-2 \pi i \theta} b^{*} a & \text { noncommutative-torus relations. }
\end{array}
$$

Here $p, q$ and $\theta$ are parameters running the unit interval $[0,1]$.

It turns out to be very helpful to distinguish the following elements of this algebra:

$$
A:=1-a a^{*} \text { and } B:=1-b b^{*} .
$$

They satisfy $A B=0$ and one can think of them as some key functions supported, respectively, on one or the other noncommutative solid torus whose glueing yields our quantum 3-sphere. It is also immediate that we have the following relations:

$$
A b=b A, \quad B a=a B, \quad A a=p a A, \quad B b=q b B, \quad a^{*} a=1-p A, \quad b^{*} b=1-q B .
$$

To prove the existence of the enveloping $C^{*}$-algebra of $\mathcal{O}\left(S_{p q \theta}^{3}\right)$, we first need to study bounded $*$-representations of the latter. It is straightforward to verify that for all values of parameters $p, q$ and $\theta$ we have: 
Proposition 2.2. Let $\left\{e_{k}\right\}_{k \in \mathbb{N}}$ and $\left\{e_{m, n}\right\}_{m \in \mathbb{Z}, n \in \mathbb{N}}$ be orthonormal bases of two Hilbert spaces, respectively. Put $\mu:=e^{2 \pi i \theta}$. Then the following formulas define bounded $*$-representations of $\mathcal{O}\left(S_{p q \theta}^{3}\right)$ :

$$
\begin{aligned}
& \text { (1) } \rho_{\lambda}(a) e_{k}=\sqrt{1-p^{k+1}} e_{k+1}, \quad \rho_{\lambda}(b) e_{k}=\lambda \mu^{-k} e_{k}, \quad \lambda \in U(1) \\
& \text { (2) } \rho_{\lambda}^{\prime}(a) e_{k}=\lambda \mu^{k} e_{k}, \quad \rho_{\lambda}^{\prime}(b) e_{k}=\sqrt{1-q^{k+1}} e_{k+1}, \quad \lambda \in U(1) \\
& \text { (3) } \rho(a) e_{m, n}=\mu^{m} \sqrt{1-p^{n+1}} e_{m, n+1}, \quad \rho(b) e_{m, n}=e_{m+1, n} \\
& \text { (4) } \rho^{\prime}(a) e_{m, n}=e_{m+1, n}, \quad \rho^{\prime}(b) e_{m, n}=\mu^{-m} \sqrt{1-q^{n+1}} e_{m, n+1}
\end{aligned}
$$

In order to check the relations, we need explicit formulas for the adjoints of the aforelisted operators. They are as follows.

$$
\begin{gathered}
\rho_{\lambda}\left(a^{*}\right) e_{k}=\left\{\begin{array}{cc}
\sqrt{1-p^{k}} e_{k-1} & \begin{array}{l}
\text { for } k>0 \\
\text { for } k=0
\end{array}
\end{array}, \quad \rho_{\lambda}\left(b^{*}\right) e_{k}=\lambda^{-1} \mu^{k} e_{k},\right. \\
\rho_{\lambda}^{\prime}\left(a^{*}\right) e_{k}=\lambda^{-1} \mu^{-k} e_{k}, \quad \rho_{\lambda}^{\prime}\left(b^{*}\right) e_{k}=\left\{\begin{array}{cc}
\sqrt{1-q^{k}} e_{k-1} & \text { for } k>0 \\
0 & \text { for } k=0
\end{array}\right. \\
\rho\left(a^{*}\right) e_{m, n}=\left\{\begin{array}{cc}
\mu^{-m} \sqrt{1-p^{n}} e_{m, n-1} & \text { for } n>0 \\
0 & \text { for } n=0
\end{array} \quad \begin{array}{l}
\rho\left(b^{*}\right) e_{m, n}=e_{m-1, n} \\
\rho^{\prime}\left(b^{*}\right) e_{m, n}=\left\{\begin{array}{cc}
\mu^{m} \sqrt{1-q^{n}} e_{m, n-1} & \text { for } n>0 \\
0 & \text { for } n=0
\end{array} .\right.
\end{array}\right.
\end{gathered}
$$

With these formulas at hand, one immediately computes:

$$
\begin{aligned}
& \rho_{\lambda}(A) e_{k}=\left\{\begin{array}{cc}
p^{k} e_{k} & \text { for } k>0 \\
e_{k} & \text { for } k=0
\end{array},\right. \\
& \rho_{\lambda}^{\prime}(A)=0, \quad \rho_{\lambda}(B)=0, \\
& \rho_{\lambda}^{\prime}(B) e_{k}=\left\{\begin{array}{cc}
q^{k} e_{k} & \text { for } k>0 \\
e_{k} & \text { for } k=0
\end{array},\right. \\
& \rho(A) e_{m, n}=\left\{\begin{array}{cc}
p^{n} e_{m, n} & \text { for } n>0 \\
e_{m, n} & \text { for } n=0
\end{array}, \quad \rho(B)=0,\right. \\
& \rho^{\prime}(A)=0, \quad \rho^{\prime}(B) e_{m, n}=\left\{\begin{array}{cc}
q^{n} e_{m, n} & \text { for } n>0 \\
e_{m, n} & \text { for } n=0
\end{array} .\right.
\end{aligned}
$$


Note now that, for $0 \leq p, q<1$, the relations (2.2) entail that the norm of $a$ and $b$ in any bounded $*$-representation $\varrho$ is at most 1 . Indeed, we have

$$
1-p=\left\|\varrho\left(a^{*} a-p a a^{*}\right)\right\| \geq\left\|\varrho\left(a^{*} a\right)\right\|-p\left\|\varrho\left(a a^{*}\right)\right\|=(1-p)\|\varrho(a)\|^{2},
$$

which implies $\|\varrho(a)\| \leq 1$. The same reasoning works for $\|\varrho(b)\|$. Hence, as the space of bounded *-representations of $\mathcal{O}\left(S_{p q \theta}^{3}\right)$ is non-empty and the generators are uniformly bounded in all of them, there exists the universal enveloping $C^{*}$-algebra of $\mathcal{O}\left(S_{p q \theta}^{3}\right)$.

Definition 2.3. For $0 \leq p, q<1$, the $C^{*}$-algebra of $S_{p q \theta}^{3}$ is the universal enveloping $C^{*}$-algebra of $\mathcal{O}\left(S_{p q \theta}^{3}\right)$. We denote it by $C\left(S_{p q \theta}^{3}\right)$.

To finish the discussion of the norm of the generators, observe that the formulas Proposition 2.2(3) entail $\|\rho(a)\|,\|\rho(b)\| \geq 1$. This fact combined with the above shown inequalities $\|\varrho(a)\|,\|\varrho(b)\| \leq 1$, for any $\varrho$, implies that

$$
\|a\|=1=\|b\|, \quad \forall 0 \leq p, q<1 .
$$

We skip the analysis of the irreducibility of the above representations and postpone the discussion of what happens for special values of parameters $p, q$ and $\theta$ for later on. Instead, we focus on the representations $\rho$ and $\rho^{\prime}$ and find a linear basis of $\mathcal{O}\left(S_{p q \theta}^{3}\right)$ for two cases of parameter values. Contrary to representation formulas, basis expressions are sensitive to special parameter values. To write basis elements, it is convenient to adopt the following convention:

$$
x_{\alpha}=\left\{\begin{array}{ccc}
x^{\alpha} & \text { for } & \alpha \in \mathbb{N} \\
\left(x^{*}\right)^{-\alpha} & \text { for } & \alpha \in \mathbb{Z} \backslash \mathbb{N} .
\end{array}\right.
$$

Lemma 2.4. If $0<p, q<1$, then the elements $a_{\alpha} b_{\beta} A^{m}, a_{\alpha^{\prime}} b_{\beta^{\prime}} B^{m^{\prime}}, \alpha, \alpha^{\prime}, \beta, \beta^{\prime}, m, m^{\prime} \in \mathbb{Z}$, $m \geq 0, m^{\prime}>0$, form a linear basis of $\mathcal{O}\left(S_{p q \theta}^{3}\right)$, and the representation $\rho \oplus \rho^{\prime}$ is faithful.

Proof. It is a straightforward consequence of relations in the algebra $\mathcal{O}\left(S_{p q \theta}^{3}\right)$ that the aforelisted elements span this algebra. Now, let

$$
f:=\sum_{\alpha, \beta \in \mathbb{Z}, l \in \mathbb{N}} f_{\alpha \beta l} a_{\alpha} b_{\beta} A^{l}+\sum_{\alpha, \beta \in \mathbb{Z}, l>0} g_{\alpha \beta l} a_{\alpha} b_{\beta} B^{l}
$$

be an arbitrary linear combination, and assume that it is equal to 0 . Then $\rho(f) e_{m, n}=0$ for any $m \in \mathbb{Z}, n \in \mathbb{N}$. On the other hand,

$$
\rho(f) e_{m, n}=\sum_{\alpha, \beta \in \mathbb{Z}, l \in \mathbb{N}} f_{\alpha \beta l} p^{n l} \mu^{m \alpha} \begin{cases}\sqrt{\left(1-p^{n+1}\right) \cdots\left(1-p^{n+\alpha}\right)} e_{m+\beta, n+\alpha} & \text { for } \quad \alpha>0 \\ e_{m+\beta, n+\alpha} & \text { for } \quad \alpha=0 \\ \sqrt{\left(1-p^{n}\right) \cdots\left(1-p^{n+\alpha+1}\right)} e_{m+\beta, n+\alpha} & \text { for } \quad \alpha<0\end{cases}
$$


Since $f$ is a finite linear combination, there exists $\alpha_{0}=\min \left\{\alpha \in \mathbb{Z} \mid f_{\alpha \beta l} \neq 0, \beta \in \mathbb{Z}, l \in \mathbb{N}\right\}$. Hence, the square roots in (2.18) are non-zero for all $n \geq-\alpha_{0}$. Therefore, it follows from the linear independence of the basis vectors that

$$
\sum_{l \in \mathbb{N}} f_{\alpha \beta l}\left(p^{n}\right)^{l}=0, \quad \forall \alpha, \beta \in \mathbb{Z}, n \geq-\alpha_{0}
$$

Thus $f_{\alpha \beta l}$ are coefficients of a polynomial vanishing at infinitely many points. Consequently $f_{\alpha \beta l}=0$ for all $\alpha, \beta, l$.

Applying now $\rho^{\prime}(f)$ to $e_{m, n}$ we obtain

$$
\rho^{\prime}(f) e_{m, n}=\sum_{\alpha, \beta \in \mathbb{Z}, l \in \mathbb{N}} g_{\alpha \beta l} q^{n l} \mu^{-m \beta}\left\{\begin{array}{ll}
\sqrt{\left(1-q^{n+1}\right) \cdots\left(1-q^{n+\beta}\right)} e_{m+\alpha, n+\beta} & \text { for } \beta>0 \\
e_{m+\alpha, n+\beta} & \text { for } \beta=0 \\
\sqrt{\left(1-q^{n}\right) \cdots\left(1-q^{n+\beta+1}\right)} e_{m+\alpha, n+\beta} & \text { for } \beta<0
\end{array} .\right.
$$

Using the same argument as above, we can conclude that, for all sufficiently big $n \in \mathbb{N}$ and for all $\alpha, \beta \in \mathbb{Z}$,

$$
\sum_{l>0} g_{\alpha \beta l}\left(q^{n}\right)^{l}=0
$$

Again as above, this entails the vanishing of all $g_{\alpha \beta l}$.

Finally, let us note that we have shown that $\left(\rho \oplus \rho^{\prime}\right)(f)=0$ implies $f=0$. This means the desired faithfulness of $\rho \oplus \rho^{\prime}$.

REMARK 2.5. Let $z$ be the generator of $\mathcal{O}\left(D_{q}\right)$ (see (1.10)). Assume the convention (2.16), and put $Z:=1-z z^{*}$. For $0<q<1$, reasoning much as in the proof of the above lemma, one can show that the elements $z_{\alpha} Z^{k}, \alpha \in \mathbb{Z}, k \in \mathbb{N}$, form a basis of $\mathcal{O}\left(D_{q}\right)$.

Let us now pass to considering the case $p=0=q$. Our main point here is that, for $0 \leq p, q<1$, we have $C\left(S_{p q \theta}^{3}\right) \cong C\left(S_{00 \theta}^{3}\right)$. To avoid confusion, we shall denote the generators of $\mathcal{O}\left(S_{00 \theta}^{3}\right)$ by $s$ and $t$, respectively, rather than by $a$ and $b$. For the time being, the latter notation is reserved for the cases $0<p, q<1$. For $p=0=q$ the relations (2.2) become simpler and the elements $A$ and $B(2.4)$ become projections. Hence, it is clear that their powers can no longer be useful in writing down basis elements. Therefore, we introduce the following self-adjoint elements:

$$
A_{k}=1-s^{k} s^{* k}, \quad B_{k}=1-t^{k} t^{* k}, \quad k \in \mathbb{N} .
$$

For these elements, one can immediately derive the identities:

$$
\begin{array}{r}
{\left[t, A_{k}\right]=0, \quad A_{k+1} s=s A_{k}, \quad s^{*} A_{k+1}=A_{k} s^{*}, \quad A_{k+1}=s A_{k} s^{*}+A_{1},} \\
{\left[s, B_{k}\right]=0, \quad B_{k+1} t=t B_{k}, \quad t^{*} B_{k+1}=B_{k} t^{*}, \quad B_{k+1}=t B_{k} t^{*}+B_{1} .}
\end{array}
$$


With this notation, we can write the sphere relation (2.1) as $A_{1} B_{1}=0$. Now, combining the first formula of (2.24) and the last of (2.23) with the standard induction proves that $A_{k} B_{1}=0$, $\forall k \in \mathbb{N}$. Applying the standard induction with this formula as the starting point, and the first formula of (2.23) and the last of (2.24) for the induction step, yields

$$
A_{k} B_{l}=0, \quad \forall k, l \in \mathbb{N} .
$$

Again adopting the convention (2.16), we can now claim:

Lemma 2.6. Let $\alpha, \beta, k \in \mathbb{Z}$. The elements $s_{\alpha} t_{\beta}$, and $s_{\alpha} t_{\beta} A_{k}$ with $k>0, \alpha>-k$, and $s_{\alpha} t_{\beta} B_{k}$ with $k>0, \beta>-k$, form a linear basis of $\mathcal{O}\left(S_{00 \theta}^{3}\right)$. The representation $\rho \oplus \rho^{\prime}$ is faithful.

Proof. It follows directly from relations in $\mathcal{O}\left(S_{00 \theta}^{3}\right)$ that the monomials $s_{\mu} t^{k} t^{* l}, \mu \in \mathbb{Z}$, $k, l \in \mathbb{N}$, and $s^{m} s^{* n} t_{\nu}, \nu \in \mathbb{Z}, m, n \in \mathbb{N}$, span $\mathcal{O}\left(S_{00 \theta}^{3}\right)$. We now want to show that so do the elements listed in the assertion of the lemma. Using (2.24), for $l>k>0$, we obtain

$$
s_{\mu} t^{k} t^{* l}=s_{\mu} t^{k} t^{* k} t^{* l-k}=s_{\mu}\left(1-B_{k}\right) t^{* l-k}=s_{\mu} t^{* l-k}-s_{\mu} t^{* l-k} B_{l} .
$$

Analogously, for $k \geq l$, we have

$$
s_{\mu} t^{k} t^{* l}=s_{\mu} t^{k-l} t^{l} t^{* l}=s_{\mu} t^{k-l}\left(1-B_{l}\right)=s_{\mu} t^{k-l}-s_{\mu} t^{k-l} B_{l} .
$$

Similarly, taking an advantage of (2.23) one checks easily for $m \geq n$ that

$$
s^{m} s^{* n} t_{\nu}=s^{m-n} s^{n} s^{* n} t_{\nu}=s^{m-n} t_{\nu}-s^{m-n} t_{\nu} A_{n} .
$$

For $n>m>0$, it follows from (2.23) that

$$
s^{m} s^{* n} t_{\nu}=s^{m} s^{* m} s^{* n-m} t_{\nu}=s^{* n-m} t_{\nu}-s^{* n-m} t_{\nu} A_{n} .
$$

Summarizing, we have shown that the elements given in the assertion of the lemma span $\mathcal{O}\left(S_{00 \theta}^{3}\right)$.

Let us now write a general element $f \in \mathcal{O}\left(S_{00 \theta}^{3}\right)$ as a linear combination:

$$
f:=\sum_{\substack{\alpha, \beta, k \in \mathbb{Z} \\ \alpha>-k<0}} f_{\alpha \beta k} s_{\alpha} t_{\beta} A_{k}+\sum_{\substack{\alpha, \beta, k \in \mathbb{Z} \\ \alpha>-k<0}} g_{\alpha \beta k} s_{\alpha} t_{\beta} B_{k}+\sum_{\alpha, \beta \in \mathbb{Z}} h_{\alpha, \beta} s_{\alpha} t_{\beta} .
$$

Using the straightforward to verify formulas

$$
\begin{aligned}
& \rho\left(A_{k}\right) e_{m, n}=\left\{\begin{array}{cc}
e_{m, n} & \text { for } k>n \\
0 & \text { for } k \leq n
\end{array}, \quad \rho\left(B_{k}\right) e_{m, n}=0,\right. \\
& \rho^{\prime}\left(A_{k}\right) e_{m, n}=0, \quad \rho^{\prime}\left(B_{k}\right) e_{m, n}=\left\{\begin{array}{cc}
e_{m, n} & \text { for } k>n \\
0 & \text { for } k \leq n
\end{array},\right.
\end{aligned}
$$

we can compute the operators $\rho(f)$ and $\rho^{\prime}(f)$. For any $m \in \mathbb{Z}$ and $n \in \mathbb{N}$, employing the shorthand notation $\mu:=e^{2 \pi i \theta}$, we have

$$
\begin{aligned}
& \rho\left(\sum_{\substack{\alpha, \beta, k \in \mathbb{Z} \\
\alpha>-k<0}} f_{\alpha \beta k} s_{\alpha} t_{\beta} A_{k}+\sum_{\substack{\alpha, \beta, k \in \mathbb{Z} \\
\beta>-k<0}} g_{\alpha \beta k} s_{\alpha} t_{\beta} B_{k}+\sum_{\alpha, \beta \in \mathbb{Z}} h_{\alpha, \beta} s_{\alpha} t_{\beta}\right) e_{m, n} \\
& =\sum_{\substack{\alpha, \beta, k \in \mathbb{Z} \\
-k<-n \leq \alpha}} f_{\alpha \beta k} \mu^{(m+\beta) \alpha} e_{m+\beta, n+\alpha}+\sum_{\substack{\alpha, \beta \in \mathbb{Z} \\
\alpha \geq-n}} h_{\alpha \beta} \mu^{(m+\beta) \alpha} e_{m+\beta, n+\alpha} .
\end{aligned}
$$


To prove the linear independence of our linear generators, we assume now that $f=0$. We begin by drawing conclusions from the entailed equality $\rho(f)=0$. If the first sum in (2.33) is not vanishing form the start, then there exists

$$
k_{0}:=\max \left\{k \in \mathbb{Z} \mid f_{\alpha \beta k} \neq 0, \alpha, \beta \in \mathbb{Z}, \alpha>-k<0\right\},
$$

and choosing $n \geq k_{0}$ makes it zero. Thus the first sum is zero at least for all sufficiently big $n$, and we obtain

$$
\sum_{\substack{\alpha, \beta \in \mathbb{Z} \\ \alpha \geq-n}} h_{\alpha \beta} \mu^{(m+\beta) \alpha} e_{m+\beta, n+\alpha}=0 .
$$

Again for all sufficiently big $n$, the condition $\alpha \geq-n$ in the foregoing sum is automatically satisfied for all possibly non-vanishing coefficients $h_{\alpha \beta}$ that appear therein. Thus we can conclude from the linear independence of the vectors $e_{m+\beta, n+\alpha}$ that

$$
h_{\alpha \beta}=0, \quad \forall \alpha, \beta \in \mathbb{Z} .
$$

Putting this back into (2.33) and noticing that terms with different $\beta$ are linearly independent, we get

$$
\sum_{\substack{\alpha, k \in \mathbb{Z} \\-k<-n \leq \alpha}} f_{\alpha \beta k} \mu^{(m+\beta) \alpha} e_{m+\beta, n+\alpha}=0, \quad \forall \beta, m \in \mathbb{Z}, n \in \mathbb{N} .
$$

Suppose now that at least one of the coefficients $f_{\alpha \beta k}$ is non-zero. Then $k_{0}$ exists. Due to the assumption that $k$ is always a positive natural number, $k_{0}-1 \geq 0$, so that we can choose $n=k_{0}-1$. Then the condition $k>k_{0}-1$ in (2.37) entails that $k_{0}$ is the only possible value for $k$, and that there is no summation over $k$. What remains is the equality

$$
\sum_{\mathbb{Z} \ni \alpha>-k_{0}} f_{\alpha \beta k_{0}} \mu^{(m+\beta) \alpha} e_{m+\beta, k_{0}-1+\alpha}=0, \quad \forall m, \beta \in \mathbb{Z} .
$$

Now the linear independence of all the summands implies that

$$
f_{\alpha \beta k_{0}}=0, \quad \forall \alpha, \beta \in \mathbb{Z}, \alpha>-k_{0} .
$$

As $\alpha$ is always assumed to be bigger than $-k$, the condition $\alpha>-k_{0}$ is automatically satisfied. This means that for all possible $\alpha$ and $\beta$ we have $f_{\alpha \beta k_{0}}=0$, which contradicts the definition of $k_{0}$. Consequently, all coefficients $f_{\alpha \beta k}$ must vanish, as desired. Thus $\rho(f)=0$ leads to the vanishing of all coefficients $f_{\alpha \beta k}$ and $h_{\alpha \beta}$. Combining it with $\rho^{\prime}(f)=0$ yields

$$
\sum_{\substack{\alpha, \beta, k \in \mathbb{Z} \\ \beta \geq-n>-k}} g_{\alpha \beta k} \mu^{-m \beta} e_{m+\alpha, n+\beta}=0, \quad \forall m \in \mathbb{Z}, n \in \mathbb{N} .
$$

Much as before, we can deduce from here that also all coefficients $g_{\alpha \beta k}$ are zero. This proves the desired linear independence.

The second assertion of the lemma follows from the observation that in the above reasoning the vanishing of all coefficients, which entails the equality $f=0$, is concluded solely from the formula $\left(\rho \oplus \rho^{\prime}\right)(f)=0$.

This way we have shown the faithfulness of $\rho \oplus \rho^{\prime}$ for any $0 \leq p, q<1$. On the other hand, it is a general fact that, if $C$ is the universal $C^{*}$-algebra of a $*$-algebra $\mathcal{O}$, and the latter admits 
a faithful bounded $*$-representation, then $\mathcal{O} \subseteq C$. Indeed, by the universality of $C$ we have the commutative diagram

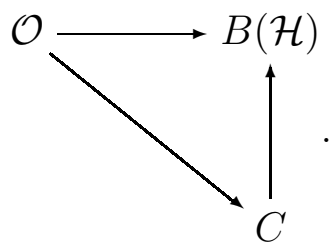

Hence, from the injectivity of the horizontal map (faithfulness of a representation), we can infer the injectivity of the diagonal map, as needed. Consequently, we can claim:

$$
\mathcal{O}\left(S_{p q \theta}^{3}\right) \subseteq C\left(S_{p q \theta}^{3}\right), \quad \forall 0 \leq p, q<1
$$

Any representation can be extended by continuity to the universal $C^{*}$-algebra, but in general there is no reason to expect that the extension of a faithful representation remains faithful. In particular, we cannot automatically claim that the extension of $\rho \oplus \rho^{\prime}$ is faithful. Here this difficulty is more pronounced as there is a problem with classifying irreducible representations, which is often a standard way for grasping the size of the universal $C^{*}$-algebra. (Recall that, in a sense, the irreducible representations play the role of points of the underlying quantum space.) Here a partial remedy to this problem is as follows.

Lemma 2.7. Let $C_{A, p}$ and $C_{B, q}$ be the $C^{*}$-subalgebras of $C\left(S_{p q \theta}^{3}\right), 0 \leq p, q<1$, generated by a and $b$, respectively. The extension of $\rho$ restricted to $C_{A, p}$ and the extension of $\rho^{\prime}$ restricted to $C_{B, q}$ are faithful.

Proof. Let us denote the aforementioned restrictions by $\rho_{A, p}$ and $\rho_{B, q}^{\prime}$, respectively. Due to the universality of the Topelitz algebra, the assignment $z \mapsto a$ defines a $C^{*}$-homomorphism $\mathcal{T} \cong C\left(D_{p}\right) \stackrel{p r}{\rightarrow} C_{A, p}$ (see (1.10)). Composing this map with $\rho_{A, p}$ yields a representation of $C\left(D_{p}\right)$ which splits into a direct sum of representations indexed by the integers corresponding to the first label of the basis $\left\{e_{m, n}\right\}_{m \in \mathbb{Z}, n \in \mathbb{N}}$. It follows from Proposition 2.2(3) that the direct summand representation corresponding to $m=0$ coincides with the extension to $C\left(D_{p}\right)$ of the representation $\pi$ in (1.11):

$$
\rho_{A, p}(p r(z)) e_{0, n}=\sqrt{1-p^{n+1}} e_{0, n+1}
$$

Since this extension is known to be faithful and $p r$ is evidently surjective, we can conclude that $\rho_{A, p}$ is injective, as needed. The reasoning for $\rho_{B, q}^{\prime}$ is identical.

The argument proving the lemma shows also the injectivity of $p r$ and its $B, q$-counterpart. This way, as both these maps are evidently surjective, we obtain as an immediate corollary that

$$
C_{A, p} \cong \mathcal{T} \cong B_{B, q}, \quad \forall p, q \in[0,1[
$$

We are now ready to proceed to the key part of this section. It employs the just proved Lemma 2.7 and is pivotal in the final $K$-theory computation.

Theorem 2.8. The $C^{*}$-algebra $C\left(S_{p q \theta}^{3}\right), 0 \leq p, q<1$, and $C\left(S_{00 \theta}^{3}\right)$ are isomorphic. 
Proof. Assume first that $0<p, q<1$. Recall our convention that $a, b$ and $s, t$ stand for the generators of $C\left(S_{p q \theta}^{3}\right)$ with $0<p, q<1$, and $C\left(S_{00 \theta}^{3}\right)$, respectively. Since $s^{*} s=1=t^{*} t$ entails $\|s\|=1=\|t\|$, one can easily verify that the infinite series

$$
\sum_{k=0}^{\infty}\left(\sqrt{1-p^{k+1}}-\sqrt{1-p^{k}}\right) s^{k+1} s^{* k} \text { and } \sum_{k=0}^{\infty}\left(\sqrt{1-q^{k+1}}-\sqrt{1-q^{k}}\right) t^{k+1} t^{* k}
$$

converge in norm to elements of $C\left(S_{00 \theta}^{3}\right)$. We denote them by $f(a)$ and $f(b)$, respectively. On the other hand, (2.2) immediately implies the positivity of the absolute values of generators: $|a|,|b|>0$. Hence $|a|,|b|$ are invertible, and we can define the following elements of $C\left(S_{p q \theta}^{3}\right)$ :

$$
g(s):=a|a|^{-1}, \quad g(t):=b|b|^{-1} .
$$

The point is to extend the formulas (2.45) and (2.46) to mutually inverse $C^{*}$-homomorphisms. Our first step is to show the existence of $f$ and $g$. Due to the universality of both $C^{*}$-algebras, it suffices to show that the elements in (2.45) and (2.46) satisfy appropriate relations.

We begin with the sphere relation for the elements in (2.46). First, we have

$$
1-g(s) g(s)^{*}=1-a|a|^{-2} a^{*}
$$

and

$$
\left(1-a|a|^{-2} a^{*}\right)\left(1-a a^{*}\right)=1-a a^{*}-a|a|^{-2} a^{*}+a\left(a^{*} a\right)^{-1} a^{*} a a^{*}=1-a|a|^{-2} a^{*} .
$$

In the same way, we obtain

$$
1-g(t) g(t)^{*}=1-b|b|^{-2} b^{*}=\left(1-b b^{*}\right)\left(1-b|b|^{-2} b^{*}\right) .
$$

Combining these facts, we compute

$$
\left(1-g(s) g(s)^{*}\right)\left(1-g(t) g(t)^{*}\right)=\left(1-a|a|^{-2} a^{*}\right)\left(1-a a^{*}\right)\left(1-b b^{*}\right)\left(1-b|b|^{-2} b^{*}\right)=0 .
$$

Here the last step follows immediately from (2.1). Next, we check the disc relations:

$$
g(s)^{*} g(s)=|a|^{-1} a^{*} a|a|^{-1}=|a|^{-2} a^{*} a=1, \quad g(t)^{*} g(t)=|b|^{-1} b^{*} b|b|^{-1}=|b|^{-2} b^{*} b=1 .
$$

Finally, we employ (2.3) for the torus relations to obtain

$$
g(s) g(t)=a|a|^{-1} b|b|^{-1}=e^{2 \pi i \theta} b a|a|^{-1}|b|^{-1}=e^{2 \pi i \theta} b|b|^{-1} a|a|^{-1}=e^{2 \pi i \theta} g(t) g(s),
$$

and, analogously, $g(s) g(t)^{*}=e^{-2 \pi i \theta} g(t)^{*} g(s)$. Thus we have shown that the formulas (2.46) define a $C^{*}$-homomorphism $g: C\left(S_{00 \theta}^{3}\right) \rightarrow C\left(S_{p q \theta}^{3}\right)$.

We now proceed to show that (2.45) defines $f$. To make easier writing down computations, let us use the notation

$$
p_{k}:=\sqrt{1-p^{k+1}}-\sqrt{1-p^{k}}, \quad q_{k}:=\sqrt{1-q^{k+1}}-\sqrt{1-q^{k}}, \quad k \in \mathbb{N}
$$


It is clear that $\sum_{k=0}^{\infty} p_{k}=1=\sum_{k=0}^{\infty} q_{k}$, and, consequently, that $\sum_{k, l=0}^{\infty} p_{k} p_{l}=1=\sum_{k, l=0}^{\infty} q_{k} q_{l}$. Hence we can write

$$
\left(1-f(a) f(a)^{*}\right)\left(1-f(b) f(b)^{*}\right)=\sum_{k, l, m, n=0}^{\infty} p_{k} p_{l} q_{m} q_{n}\left(1-s^{k+1} s^{* k} s^{l} s^{* l+1}\right)\left(1-t^{m+1} t^{* m} t^{n} t^{* n+1}\right) .
$$

It follows from $s^{*} s=1=t^{*} t$ that the terms in parentheses are always of the form $A_{i}$ and $B_{j}$, respectively. Thus the formula (2.25) entails the desired equality

$$
\left(1-f(a) f(a)^{*}\right)\left(1-f(b) f(b)^{*}\right)=0 .
$$

Next, using $s t=e^{2 \pi i \theta} t s$ and $s t^{*}=e^{-2 \pi i \theta} t^{*} s$ we compute

$$
f(a) f(b)=\sum_{k, l=0}^{\infty} p_{k} q_{l} s^{k+1} s^{* k} t^{l+1} t^{* l}=e^{2 \pi i \theta} \sum_{k, l=0}^{\infty} p_{k} q_{l} t^{l+1} t^{* l} s^{k+1} s^{* k}=e^{2 \pi i \theta} f(b) f(a)
$$

and, similarly,

$$
f(a) f(b)^{*}=e^{-2 \pi i \theta} f(b)^{*} f(a) .
$$

For the remaining disc relations, we resort to the help of available representations. Using definitions from Lemma 2.7, it is straightforward to check that

$$
\rho_{A, 0}(f(a))=\rho_{A, p}(a), \quad \rho_{B, 0}^{\prime}(f(b))=\rho_{B, q}^{\prime}(b), \quad \rho_{A, p}(g(s))=\rho_{A, 0}(s), \quad \rho_{B, q}^{\prime}(g(t))=\rho_{B, 0}^{\prime}(t) .
$$

Therefore, since $\rho_{A, 0}$ and $\rho_{B, 0}^{\prime}$ are injective and

$$
f(a)^{*} f(a)-p f(a) f(a)^{*}-1+p \in C_{A, 0}, \quad f(b)^{*} f(b)-q f(b) f(b)^{*}-1+q \in C_{B, 0},
$$

the disc relations (2.2) entail that

$$
f(a)^{*} f(a)=p f(a) f(a)^{*}+1-p, \quad f(b)^{*} f(b)=q f(b) f(b)^{*}+1-q,
$$

as needed. Hence the formulas (2.45) define a $C^{*}$-homomorphism $f: C\left(S_{p q \theta}^{3}\right) \rightarrow C\left(S_{00 \theta}^{3}\right)$.

It remains to show that $f$ and $g$ are mutually inverse. To this end, we shall again take advantage of representations. First, note that the formulas (2.57) immediately imply

$$
\rho_{A, p}=\left.\rho_{A, 0} \circ f\right|_{C_{A, p}}, \quad \rho_{B, q}^{\prime}=\left.\rho_{B, 0}^{\prime} \circ f\right|_{C_{B, q}}, \quad \rho_{A, 0}=\left.\rho_{A, p} \circ g\right|_{C_{A, 0}}, \quad \rho_{B, 0}^{\prime}=\left.\rho_{B, q}^{\prime} \circ g\right|_{C_{B, 0}} .
$$

Hence we obtain

$$
\begin{array}{ll}
\rho_{A, p}=\left.\left.\rho_{A, p} \circ g\right|_{C_{A, 0}} \circ f\right|_{C_{A, p}}, & \rho_{B, q}^{\prime}=\left.\left.\rho_{B, q}^{\prime} \circ g\right|_{C_{B, 0}} \circ f\right|_{C_{B, q}}, \\
\rho_{A, 0}=\left.\left.\rho_{A, 0} \circ f\right|_{C_{A, p}} \circ g\right|_{C_{A, 0}}, & \rho_{B, 0}^{\prime}=\left.\left.\rho_{B, 0}^{\prime} \circ f\right|_{C_{B, q}} \circ g\right|_{C_{B, 0}} .
\end{array}
$$

Consequently, the injectivity of all four representations used above (Lemma 2.7) implies that

$$
\begin{array}{ll}
\mathrm{id}=\left.\left.g\right|_{C_{A, 0}} \circ f\right|_{C_{A, p}}, & \text { id }=\left.\left.g\right|_{C_{B, 0}} \circ f\right|_{C_{B, q}}, \\
\mathrm{id}=\left.\left.f\right|_{C_{A, p}} \circ g\right|_{C_{A, 0}}, & \text { id }=\left.\left.f\right|_{C_{B, q}} \circ g\right|_{C_{B, 0}} .
\end{array}
$$


Therefore, as $a, b$ and $s, t$ generate $C\left(S_{p q \theta}^{3}\right)$ and $C\left(S_{00 \theta}^{3}\right)$, respectively, we can conclude that $f$ and $g$ are mutually inverse, as desired. This can be summarized by the commutative diagram

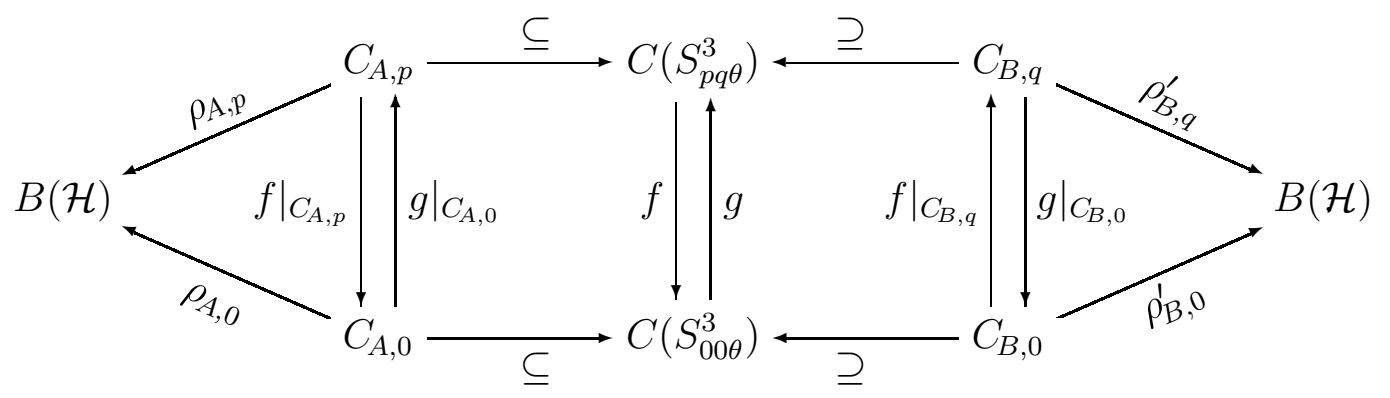

Finally, note that the formulas for $f$ and $g$ also make sense in the special cases when either $p$ or $q$ is equal to zero. Then the same argument shows that they give rise to mutually inverse $*$-homomorphisms. (For instance, if $p=0$, then the left column of the above diagram is simply given by identity maps.)

Let us finish this section by discussing some special cases. Putting $\theta=0$ but keeping $0 \leq p, q<1$ immediately recovers quantum-sphere algebras in [CM02, HMS]. On the other hand, since the norm condition (2.15) is automatically satisfied for $0 \leq p, q<1$, adding it to the definition of $C\left(S_{p q \theta}^{3}\right), 0 \leq p, q<1$, would leave it unchanged. Now, however, taking for granted the theory of commutative $C^{*}$-algebras, it is clear from (1.3) that setting in the thus defined $C^{*}$-algebra $p=1=q$ and $\theta=0$ would yield $C\left(S^{3}\right)$. This is why we view our construction as a noncommutative deformation of the topological 3-sphere. Furthermore, setting $p=1=q$ but keeping $\theta$ arbitrary gives precisely the construction of the Matsumoto (constant $\theta$ ) $C^{*}$-algebra of a noncommutative 3 -sphere [M-K91, p.334].

\section{The fiber-product $\mathrm{C}^{*}$-algebra}

The aim of this section is to prove that $C\left(S_{00 \theta}^{3}\right)$ is isomorphic to a certain fiber product (pullback) of $C^{*}$-algebras, which will make it possible to compute its $K$-groups using the MayerVietoris exact sequence.

Let $\mathcal{T}$ be the Toeplitz algebra, as defined in the preliminaries, and $z$ its generator. The crossed products $\mathcal{T} \rtimes_{\theta} \mathbb{Z}$ and $\mathcal{T} \rtimes_{-\theta} \mathbb{Z}$ with respect to the actions $\alpha^{ \pm \theta}: \mathbb{Z} \times \mathcal{T} \rightarrow \mathcal{T}$ given by $\alpha^{ \pm \theta}(n, z)=e^{ \pm 2 \pi i n \theta} z$ can be identified with the universal $C^{*}$-algebras [B-B98, p.71] generated by $s_{+}, u$, respectively $t_{-}, v$, satisfying

$$
\begin{aligned}
& s_{+}^{*} s_{+}=1=u^{*} u=u u^{*}, \quad s_{+} u=e^{2 \pi i \theta} u s_{+}, \\
& t_{-}^{*} t_{-}=1=v^{*} v=v v^{*}, \quad t_{-} v=e^{-2 \pi i \theta} v t_{-} .
\end{aligned}
$$

(Note that $s_{+} \mapsto t_{-}, u \mapsto v^{*}$ defines an isomorphism $\mathcal{T} \rtimes_{\theta} \mathbb{Z} \rightarrow \mathcal{T} \rtimes_{-\theta} \mathbb{Z}$.)

We interpret $\mathcal{T}$ as the algebra of a quantum disc, and the just defined crossed products as algebras of noncommutative solid tori. What we want to show is that our noncommutative 
3-sphere $S_{00 \theta}^{3}$ is the quotient of the disjoint union of such two quantum solid tori by an identification of their boundaries. The common boundary of the two solid tori is the noncommutative 2 -torus $T_{\theta}^{2}$ given by the (ir)rational rotation algebra explained in preliminaries. The glueing of the solid tori along their boundaries is with the exchange of fundamental cycles of the boundaries, i.e., a cycle "through a hole" is identified with a cycle "around a hole". This is reflected in the exchange of the sign of $\theta$ in the construction of the quantum solid tori.

With this picture in mind, we have natural epimorphisms

$$
\begin{array}{lll}
\pi_{1}: \mathcal{T} \underset{\theta}{\rtimes} \mathbb{Z} \longrightarrow C\left(T_{\theta}^{2}\right), & \pi_{1}\left(s_{+}\right):=x, & \pi_{1}(u):=y, \\
\pi_{2}: \mathcal{T} \underset{-\theta}{\rtimes} \mathbb{Z} \longrightarrow C\left(T_{\theta}^{2}\right), & \pi_{2}\left(t_{-}\right):=y, & \pi_{2}(v):=x .
\end{array}
$$

Here $x$ and $y$ are the unitary generators of $C\left(T_{\theta}^{2}\right)$ (see 1.12). These two maps allow us to define the desired fiber product of $\mathcal{T} \rtimes_{\theta} \mathbb{Z}$ with $\mathcal{T} \rtimes_{-\theta} \mathbb{Z}$ over $C\left(T_{\theta}^{2}\right)$ :

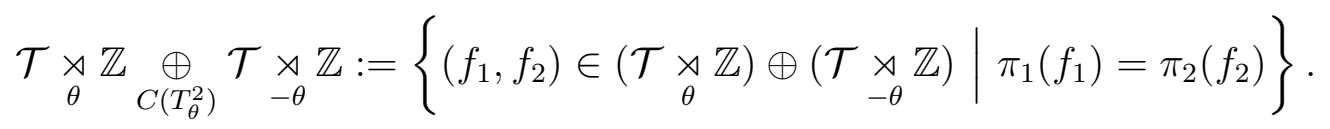

Now we can define a homomorphism

$$
h: C\left(S_{00 \theta}^{3}\right) \longrightarrow \underset{\mathcal{T}}{\underset{\theta}{\rtimes} \mathbb{Z}} \underset{C\left(T_{\theta}^{2}\right)}{\oplus} \mathcal{T} \underset{-\theta}{\rtimes} \mathbb{Z}, \quad h(s):=\left(s_{+}, v\right), \quad h(t):=\left(u, t_{-}\right) .
$$

The situation is summarized in the following commutative diagram:

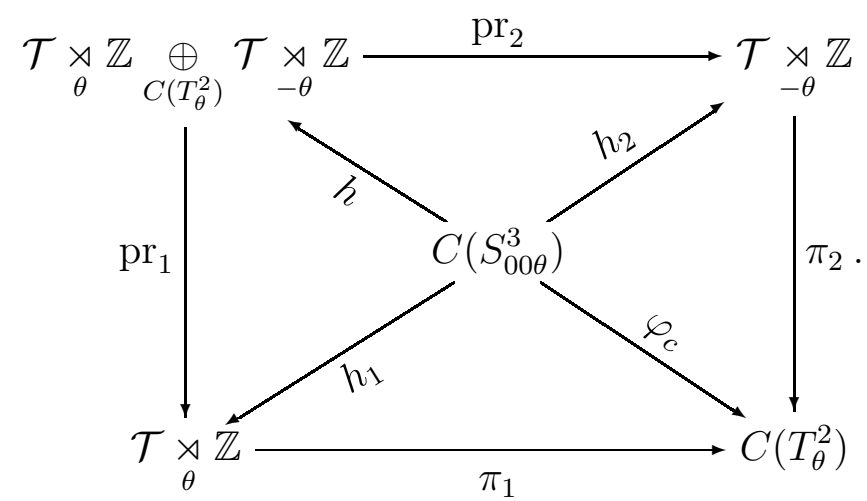

Here $\mathrm{pr}_{1}$ and $\mathrm{pr}_{2}$ are the natural projections, and $h_{1}, h_{2}, \varphi_{c}$ are the appropriate composite maps. Note that all these homomorphisms introduced above are well-defined on the $C^{*}$-level because their domains of definition are universal $C^{*}$-algebras.

Our goal is to show that $h$ is an isomorphism. To this end, we need to introduce some more $C^{*}$-homomorphisms whose existence is guaranteed by the following general result:

Lemma 3.1. Let $C$ be a (not necessarily unital) $C^{*}$-algebra, $w$ the unitary generator of the $C^{*}$-algebra of continuous functions on $S^{1}$, and $E_{i j}$ the matrices with zero everywhere except for the ij-place where there is 1 (matrix units). The assignment $E_{i j} \otimes 1 \mapsto e_{i j}, E_{i j} \otimes w \mapsto w_{i j}$, $e_{i j}, w_{i j} \in C$, defines a homomorphism $\mathcal{K} \otimes C\left(S^{1}\right) \rightarrow C$ if and only if the following conditions are satisfied for any $N \in \mathbb{N}$ :

(1) the $e_{i j}$ fulfill the relations of $E_{i j}$, i.e., $e_{i j} e_{k l}=\delta_{j k} e_{i l}, e_{i j}^{*}=e_{j i}, \forall 0 \leq i, j, k, l \leq N$; 
(2) $W:=\sum_{i=0}^{N} w_{i i}$ is a partial unitary, i.e., $W^{*} W=W W^{*}$ is a projector;

(3) $\left[W, e_{i j}\right]=0, e_{i j} W=w_{i j}, \forall 0 \leq i, j \leq N$.

Proof. Note first that for a given homomorphism $\mathcal{K} \otimes C\left(S^{1}\right) \rightarrow C$, the values on $E_{i j} \otimes 1$ and $E_{i j} \otimes w$ obviously have to satisfy the above conditions. For the proof the other way around, choose an arbitrary $N \in \mathbb{N}$ and consider $M_{N+1}(\mathbb{C}) \otimes C\left(S^{1}\right)$. To show that there exists a homomorphism from this $C^{*}$-algebra to $C$, by the universality of the tensor product ([T-M79, Proposition 4.7]), it suffices to show that there exist homomorphisms $M_{N+1}(\mathbb{C}) \rightarrow C$ and $C\left(S^{1}\right) \rightarrow C$ whose ranges commute. Since $E_{i j}$ form a basis of $M_{N+1}(\mathbb{C})$, the condition (1) guarantees that the assignment $E_{i j} \mapsto e_{i j}$ defines a $C^{*}$-algebra homomorphism. On the other hand, for any bounded normal operator $U$ such that $\left(U^{*} U\right)^{2}=U^{*} U$, we have $U=U\left(U^{*} U\right)$. Indeed, as $|U|:=\sqrt{U^{*} U}$ can be approximated by polynomials divisible by $U^{*} U$, the desired formula follows from the polar decomposition:

$$
U\left(U^{*} U\right)=\operatorname{Phase}(U)|U| U^{*} U=\operatorname{Phase}(U) \lim _{n \rightarrow \infty}\left(p_{n}\left(U^{*} U\right) U^{*} U\right) U^{*} U=U .
$$

Therefore, since every $C^{*}$-algebra admits a faithful representation, we can conclude from (2) that $W=W\left(W^{*} W\right)$. Now it follows from the normality of $W$ that $W^{*} W$ is the identity of the $C^{*}$-subalgebra generated by $W$. Hence the assignment $w \mapsto W$ defines a homomorphism $C\left(S^{1}\right) \rightarrow C$. By (3), the ranges of the two just constructed homomorphisms commute. Thus, for any $N \in \mathbb{N}$, we have a homomorphism $M_{N+1}(\mathbb{C}) \otimes C\left(S^{1}\right) \rightarrow C$. These homomorphisms define a $*$-algebra homomorphism $\bigcup_{N \in \mathbb{N}} M_{N+1}(\mathbb{C}) \otimes C\left(S^{1}\right) \rightarrow C$. Since the value of this homomorphism on any element is given by applying a $C^{*}$-homomorphism, its norm is preserved or decreased. Consequently, the *-algebra homomorphism is norm-continuous and it extends to a homomorphism:

$$
\mathcal{K} \otimes C\left(S^{1}\right)=\overline{\bigcup_{N \in \mathbb{N}} M_{N+1}(\mathbb{C}) \otimes C\left(S^{1}\right)} \stackrel{F}{\longrightarrow} C .
$$

To end the proof, it suffices to note that $F\left(E_{i j} \otimes 1\right)=e_{i j}$ and that the condition (3) entails $F\left(E_{i j} \otimes w\right)=w_{i j}$.

We are now ready to prove the main statement of this section:

Theorem 3.2. The homomorphism (3.6) is an isomorphism of the universal $C^{*}$-algebra $C\left(S_{00 \theta}^{3}\right)$ with the fiber-product $C^{*}$-algebra $\mathcal{T} \rtimes_{\theta} \mathbb{Z} \oplus_{C\left(T_{\theta}^{2}\right)} \mathcal{T} \rtimes_{-\theta} \mathbb{Z}$.

Proof. Our strategy is to construct a commutative diagram of two short exact sequences and then conclude the assertion of the theorem by the Five Isomorhisms Lemma. To begin with, one can verify with the help of Lemma 3.1 that the formulas

$$
\begin{aligned}
& \left(E_{i j} \otimes w, 0\right) \longmapsto s^{i}\left(1-s s^{*}\right) t s^{* j}, \quad\left(0, w \otimes E_{i j}\right) \longmapsto t^{i}\left(1-t t^{*}\right) s t^{* j}, \quad \text { and } \\
& \left(E_{i j} \otimes w, 0\right) \longmapsto\left(s_{+}^{i}\left(1-s_{+} s_{+}^{*}\right) u s_{+}^{* j}, 0\right), \quad\left(0, w \otimes E_{i j}\right) \longmapsto\left(0, t_{-}^{i}\left(1-t_{-} t_{-}^{*}\right) v t_{-}^{* j}\right),
\end{aligned}
$$

where $E_{i j}$ are the matrix units and $w$ is the unitary generator of $C\left(S^{1}\right)$, define homomorphisms

$$
\begin{aligned}
& j_{c}:\left(\mathcal{K} \otimes C\left(S^{1}\right)\right) \oplus\left(C\left(S^{1}\right) \otimes \mathcal{K}\right) \longrightarrow C\left(S_{00 \theta}^{3}\right) \quad \text { and } \\
& j_{d}:\left(\mathcal{K} \otimes C\left(S^{1}\right)\right) \oplus\left(C\left(S^{1}\right) \otimes \mathcal{K}\right) \longrightarrow \mathcal{T} \rtimes_{\theta} \mathbb{Z} \underset{C\left(T_{\theta}^{2}\right)}{\oplus} \mathcal{T} \rtimes_{-\theta} \mathbb{Z},
\end{aligned}
$$


respectively. Observe also that the above formulas entail

$$
\begin{aligned}
& j_{c}\left(E_{i j} \otimes 1,0\right)=s^{i}\left(1-s s^{*}\right) s^{* j}, \quad j_{c}\left(0,1 \otimes E_{i j}\right)=t^{i}\left(1-t t^{*}\right) t^{* j}, \\
& j_{d}\left(E_{i j} \otimes 1,0\right)=\left(s_{+}^{i}\left(1-s_{+} s_{+}^{*}\right) s_{+}^{* j}, 0\right), \quad j_{d}\left(0,1 \otimes E_{i j}\right)=\left(0, t_{-}^{i}\left(1-t_{-} t_{-}^{*}\right) t_{-}^{* j}\right) .
\end{aligned}
$$

Next, define $\varphi_{d}$ as the composition $\pi_{1} \circ \mathrm{pr}_{1}=\pi_{2} \circ \mathrm{pr}_{2}$ and recall that $\varphi_{c}:=\varphi_{d} \circ h$ (see (3.7)). One can immediately check the commutativity of the diagram:

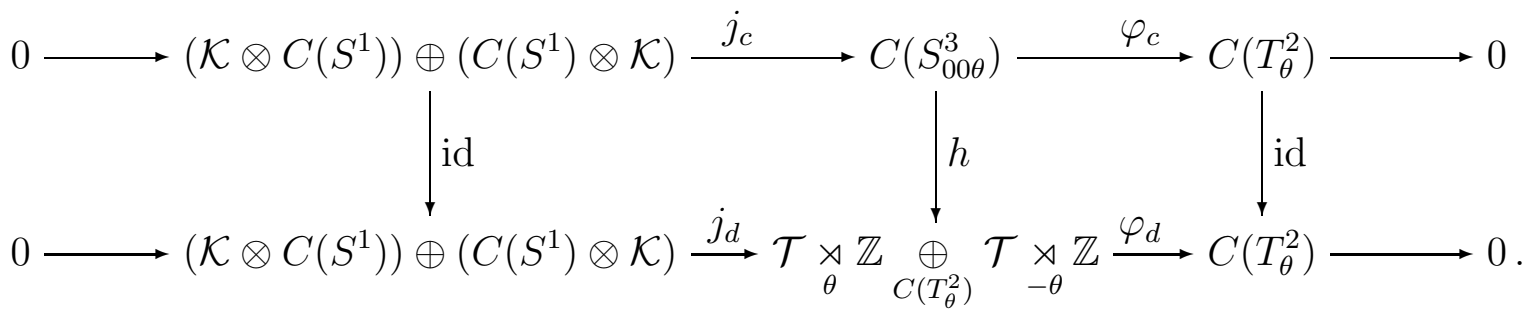

A key step is to prove the exactness of the rows. Consider first the upper row. In order to show the injectivity of $j_{c}$, we will show that $\left(\rho \oplus \rho^{\prime}\right) \circ j_{c}$ is injective. (Here $\rho$ and $\rho^{\prime}$ are representations from Proposition 2.2.) Since $\left(\rho \circ j_{c}\right)\left(0, T^{\prime}\right)=0=\left(\rho^{\prime} \circ j_{c}\right)(T, 0)$, this is the case if and only if the homomorphisms

$$
\begin{array}{ll}
\psi: \mathcal{K} \otimes C\left(S^{1}\right) \longrightarrow B(\mathcal{H}), & \psi(T):=\left(\rho \circ j_{c}\right)(T, 0), \\
\psi^{\prime}: C\left(S^{1}\right) \otimes \mathcal{K} \longrightarrow B(\mathcal{H}), & \psi^{\prime}\left(T^{\prime}\right):=\left(\rho^{\prime} \circ j_{c}\right)\left(0, T^{\prime}\right),
\end{array}
$$

are both injective. Let us check the injectivity of $\psi$. Clearly, Ker $\psi$ is a closed ideal in $\mathcal{K} \otimes C\left(S^{1}\right)$. Hence it is of the form $\mathcal{K} \otimes I$, with $I$ an ideal in $C\left(S^{1}\right)$. In particular, for any $f \in I$, we have $E_{00} \otimes f \in \operatorname{Ker} \psi$. Now one checks that

$$
\psi\left(E_{00} \otimes w\right) e_{m, n}=\delta_{0 n} e_{m+1,0}, \quad \text { i.e., } \quad \psi\left(E_{00} \otimes w\right)=\sigma_{d} \otimes E_{00},
$$

where $\sigma_{d}$ is the standard two-sided shift. Therefore, since the assignment $w \mapsto \sigma_{d}$ defines a faithful representation $\rho_{d}$ of $C\left(S^{1}\right)$, for any $f \in I$ we have:

$$
0=\psi\left(E_{00} \otimes f\right)=\rho_{d}(f) \otimes E_{00} \quad \Rightarrow \quad f=0 .
$$

Consequently, Ker $\psi=0$. One argues analogously for $\psi^{\prime}$. Thus, the injectivity of $j_{c}$ is shown. For the middle exactness, note that $\varphi_{c} \circ j_{c}=0$, so that $\varphi_{c}$ induces the map

$$
C\left(S_{00 \theta}^{3}\right) / j_{c}\left(\left(\mathcal{K} \otimes C\left(S^{1}\right)\right) \oplus\left(\mathcal{K} \otimes C\left(S^{1}\right)\right)\right) \longrightarrow C\left(T_{\theta}^{2}\right)
$$

On the other hand, it follows from (3.1-3.2) and (3.14) that the classes of $s$ and $t$ in the quotient algebra satisfy the relations (1.12) of the noncommutative torus. Therefore, due to the universality of the $C^{*}$-algebra $C\left(T_{\theta}^{2}\right)$, there is a natural homomorphism in the other direction, which is evidently the inverse of (3.21). This finishes the proof of exactness of the upper sequence.

The proof for the lower sequence proceeds along the same lines. This time, instead of $\rho$ and $\rho^{\prime}$ one uses $\bar{\rho} \circ \operatorname{pr}_{1}$ and $\bar{\rho}^{\prime} \circ \operatorname{pr}_{2}$, where $\bar{\rho}$ and $\bar{\rho}^{\prime}$ are representations of $\mathcal{T} \rtimes_{\theta} \mathbb{Z}$ and $\mathcal{T} \rtimes_{-\theta} \mathbb{Z}$, respectively, defined by

$$
\begin{aligned}
& \bar{\rho}\left(s_{+}\right) e_{m, n}=\mu^{m} e_{m, n+1}, \quad \bar{\rho}(u) e_{m, n}=e_{m+1, n} \\
& \bar{\rho}^{\prime}\left(t_{-}\right) e_{m, n}=\mu^{-m} e_{m, n+1}, \quad \bar{\rho}^{\prime}(v) e_{m, n}=e_{m+1, n} .
\end{aligned}
$$


These representations are related to $\rho$ and $\rho^{\prime}$ by the maps $h_{1}$ and $h_{2}$ of the diagram (3.7): $\rho=\bar{\rho} \circ h_{1}$ and $\rho^{\prime}=\bar{\rho}^{\prime} \circ h_{2}$. To finish the exactness proof, we identify

$$
\left(\begin{array}{c}
\mathcal{T} \underset{\theta}{\rtimes} \underset{C\left(T_{\theta}^{2}\right)}{\oplus} \underset{-\theta}{\mathcal{T}} \underset{-}{\rtimes} \mathbb{Z}
\end{array}\right) / j_{d}\left(\left(\mathcal{K} \otimes C\left(S^{1}\right)\right) \oplus\left(\mathcal{K} \otimes C\left(S^{1}\right)\right)\right)
$$

with $C\left(T_{\theta}^{2}\right)$ by analysing the classes of generators $\left(s_{+}, v\right)$ and $\left(u, t_{-}\right)$. Now, the proof of the theorem is completed by applying the Five Isomorphisms Lemma to the diagram (3.16).

One can infer from the above proof that there is a short exact sequence

$$
0 \longrightarrow \mathcal{K} \otimes C\left(S^{1}\right) \longrightarrow \underset{\theta}{\mathcal{x}} \underset{\theta}{\mathbb{Z}} \longrightarrow C\left(S^{1}\right) \underset{\theta}{\rtimes} \mathbb{Z}=C\left(T_{\theta}^{2}\right) \longrightarrow 0
$$

We can interpret it as the decomposition of our quantum solid torus into its inside and the boundary. An interesting phenomenon manifests itself in this decomposition. It makes this quantum solid torus significantly different from the one considered by Matsumoto [M-K91], notably $C\left(D^{2}\right) \rtimes_{\theta} \mathbb{Z}$. The inside of the latter torus is composed out of the nested noncommutative tori shrinking to a circle, so that the action of $\mathbb{Z}$ is not restricted to the boundary of the disc $D^{2}$. On the contrary, in our case there is no parameter $\theta$ in the ideal representing the inside of

the quantum solid torus. In this sense, the noncommutative deformation of the algebra of the interior of $D^{2}$ into $\mathcal{K}$ pushed out the $\theta$-deformation.

\section{The K-groups}

In what follows, we compute the $K$-theory of the fiber-product $C^{*}$-algebra $\mathcal{T} \rtimes_{\theta} \mathbb{Z}_{{ }_{C\left(T_{\theta}^{2}\right)}} \mathcal{T} \rtimes_{-\theta} \mathbb{Z}$ from the previous section. This, combined with identifications of this fiber product with the universal $C^{*}$-algebras of Section 2 , yields the main result of our paper, which is the computation of the $K$-groups of the Heegaard-type quantum 3-spheres.

As the first step, let us determine the $K$-theory of the crossed product $\mathcal{T} \rtimes_{\theta} \mathbb{Z} \cong \mathcal{T} \rtimes_{-\theta} \mathbb{Z}$. We know that $K_{0}(\mathcal{T}) \cong \mathbb{Z}$ (generated by the class of $1 \in \mathcal{T}$ ) and $K_{1}(\mathcal{T})=0$ [W-NE93, p.179]. Therefore, since the action of $\mathbb{Z}$ on $1 \in \mathcal{T}$ is trivial, the Pimsner-Voiculescu exact sequence [B-B98, Theorem 10.2.1] reduces to

$$
0 \longrightarrow K_{1}(\underset{\theta}{\mathcal{T} \underset{\theta}{\mathbb{Z}}}) \longrightarrow \mathbb{Z} \stackrel{0}{\longrightarrow} \mathbb{Z} \longrightarrow K_{0}(\underset{\theta}{\mathcal{T} \underset{\theta}{ } \mathbb{Z}}) \longrightarrow 0 .
$$

This immediately leads to

$$
K_{j}(\underset{ \pm \theta}{\mathcal{T} \rtimes \mathbb{Z}}) \cong \mathbb{Z}, \quad j \in\{0,1\}
$$

and gives explicitly 2 out of the 6 terms of the Mayer-Vietoris exact sequence [B-B98, Example 21.1.2(a) and Theorem 21.2.2]: 


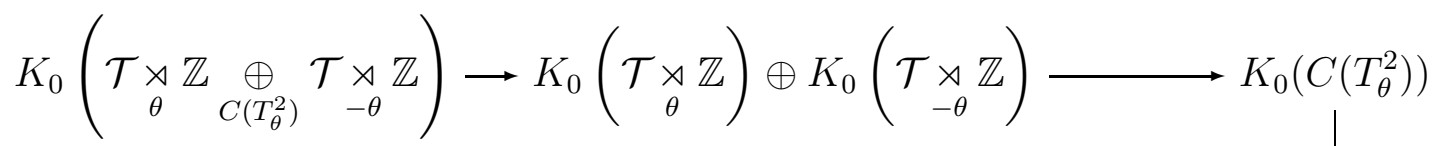

$$
\begin{aligned}
& K_{1}\left(C\left(T_{\theta}^{2}\right)\right) \longleftarrow K_{1}\left(\underset { \mathcal { T } \underset { \theta } { \mathcal { T } } \mathbb { Z } } { \longleftarrow } \oplus K _ { 1 } \left(\underset { \mathcal { T } \underset { - \theta } { \mathcal { T } } \mathbb { Z } } { \longleftarrow } \longleftarrow K _ { 1 } \left(\underset{\theta}{\left.\mathcal{T} \underset{\theta}{\mathbb{Z}} \underset{C\left(T_{\theta}^{2}\right)}{\oplus} \mathcal{T} \underset{-\theta}{\mathcal{T}} \mathbb{Z}\right)}\right.\right.\right.
\end{aligned}
$$

For further unravelling of this sequence recall that $K_{0}\left(C\left(T_{\theta}^{2}\right)\right) \cong \mathbb{Z} \oplus \mathbb{Z}[\mathrm{W}-\mathrm{NE} 93$, Section 12.3]. Here the first $\mathbb{Z}$ is generated by the unit of the $C^{*}$-algebra $C\left(T_{\theta}^{2}\right)$. The second $\mathbb{Z}$ is generated by the "Hopf line bundle" on the noncommutative torus $T_{\theta}^{2}$. Since $K_{0}\left(\mathcal{T} \rtimes_{ \pm \theta} \mathbb{Z}\right) \cong \mathbb{Z}$ and is generated by the unit of $\mathcal{T} \rtimes_{\theta} \mathbb{Z}$, the upper right horizontal arrow becomes

$$
\mathbb{Z} \oplus \mathbb{Z} \ni(m, n) \longmapsto(m-n, 0) \in \mathbb{Z} \oplus \mathbb{Z}
$$

On the other hand, $K_{1}\left(C\left(T_{\theta}^{2}\right)\right) \cong \mathbb{Z} \oplus \mathbb{Z}$, where the generators are the classes of the two unitary generators of $C\left(T_{\theta}^{2}\right)$. Therefore, since $K_{1}\left(\mathcal{T} \rtimes_{ \pm \theta} \mathbb{Z}\right) \cong \mathbb{Z}$ is generated by the unitary coming from the action of $\mathbb{Z}$, the lower left horizontal arrow becomes

$$
\mathbb{Z} \oplus \mathbb{Z} \ni(m, n) \longmapsto(m,-n) \in \mathbb{Z} \oplus \mathbb{Z} .
$$

It is evidently an isomorphism, whence the preceding and following arrows are zero maps. Summarizing, with the shorthand notation $G_{0}$ and $G_{1}$ for the left-top and right-bottom corners of (4.3), respectively, we have obtained:

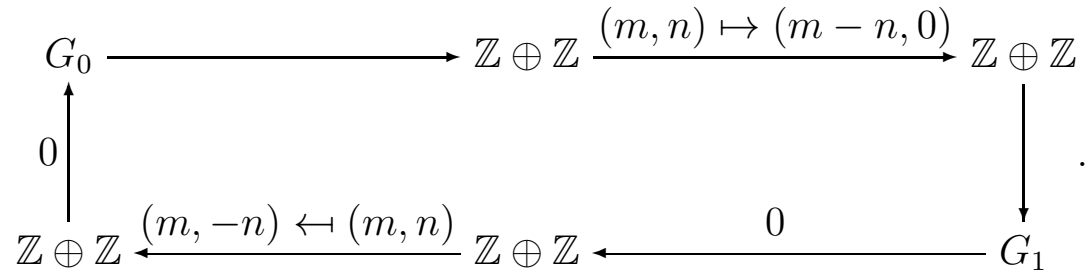

The exactness of this sequence entails that

$$
\begin{aligned}
& G_{0} \cong \operatorname{Ker}\{\mathbb{Z} \oplus \mathbb{Z} \ni(m, n) \longmapsto(m-n, 0) \in \mathbb{Z} \oplus \mathbb{Z}\} \cong \mathbb{Z} \\
& G_{1} \cong \operatorname{Coker}\{\mathbb{Z} \oplus \mathbb{Z} \ni(m, n) \longmapsto(m-n, 0) \in \mathbb{Z} \oplus \mathbb{Z}\} \cong \mathbb{Z} .
\end{aligned}
$$

Finally, combining (4.7-4.8) with Theorem 3.2 and Theorem 2.8 yields our main claim establishing the $K$-groups of the universal $C^{*}$-algebra of Definition 2.3:

Theorem 4.1. $K_{j}\left(C\left(S_{p q \theta}^{3}\right)\right) \cong \mathbb{Z}, j \in\{0,1\}, 0 \leq p, q, \theta<1$.

Acknowledgments. Much of the joint work on this paper took place in the Institut Mittag-Leffler (IML) in Djursholm and the Institut des Hautes Études Scientifiques (IHES) in Bures-sur-Yvette. It is a pleasure to thank these institutes for their wonderful hospitality and support. Here special thanks are due to C. Cheikhchoukh, F. Schmit and M.C. Vergne of IHES for their help with preparing the manuscript. P.M.H. is also grateful to S.L. Woronowicz for some $C^{*}$-algebraic inspiration, and to M. Rieffel for discussions. This work was partially supported by the KBN grant 2 P03A 01324 (P.M.H., R.M. and W.S.), IML and IHES visiting stipends (P.B. and P.M.H.), and the Graduiertenkolleg "Quantenfeldtheorie" at Universität Leipzig (P.M.H.). 


\section{References}

[AH62] Atiyah M.F., Hirzebruch F., Analytic cycles on complex manifolds. Topology 1 (1962) 25-45.

[B-PF72] Baum P.F., Lectures on K-theory, (based on lectures by F. Hirzebruch), Algebraic Topology, ed. J. F. Adams, London Math Soc. Lecture Notes Series 4, 223-238, Cambridge University Press, Cambridge, 1972.

[B-B98] Blackadar B., $K$-theory for operator algebras. Second edition. Mathematical Sciences Research Institute Publications, 5. Cambridge University Press, Cambridge, 1998.

[BEEK91] Bratteli O., Elliott G.A., Evans D.E., Kishimoto A., Noncommutative spheres. I. Internat. J. Math. 2 (1991) 139-166.

[BEEK92] Bratteli O., Elliott G.A., Evans D.E., Kishimoto A., Noncommutative spheres. II. Rational rotations. J. Operator Theory 27 (1992) 53-85.

[BK92] Bratteli O., Kishimoto A., Noncommutative spheres. III. Irrational rotations. Comm. Math. Phys. 147 (1992) 605-624.

[BHMS] Brzeziński T., Hajac P.M., Matthes R., Szymański W., The Chern character and Fredholm index for principal extensions of noncommutative algebras. In progress. Preliminary version available at http://www.fuw.edu.pl/ $\sim \mathrm{pmh}$.

[CM02] Calow D., Matthes R., Connections on locally trivial quantum principal fibre bundles. J. Geom. Phys. 41 (2002) 114-165.

[CD02] Connes A., Dubois-Violette M., Noncommutative finite-dimensional manifolds. I. Spherical manifolds and related examples. Comm. Math. Phys. 230 (2002) 539-579.

[CD03] Connes A., Dubois-Violette M., Moduli space and structure of noncommutative 3-spheres. Lett. Math. Phys. 66 (2003) 91-121.

[D-L03] Dąbrowski L., The garden of quantum spheres. Noncommutative geometry and quantum groups (Warsaw, 2001), 37-48, Banach Center Publ., 61, Polish Acad. Sci., Warsaw, 2003.

[HMS] Hajac P.M., Matthes R., Szymański W., A locally trivial quantum Hopf fibration. To appear in Algebras Representation Theory.

[K-M78] Karoubi M., K-theory. An introduction. Grundlehren der Mathematischen Wissenschaften, Band 226. Springer-Verlag, Berlin-New York, 1978.

[KL93] Klimek S., Lesniewski A., A two-parameter quantum deformation of the unit disc. J. Funct. Anal. 115 (1993) 1-23.

[M-K91] Matsumoto K., Non-commutative three-dimensional spheres, Japan J. Math. 17 (1991) 333-356. 
[N-GL97] Naber G.L., Topology, geometry, and gauge fields. Foundations. Texts in Applied Mathematics, 25, Springer-Verlag, New York, 1997.

[P-GK79] Pedersen, G.K.: $C^{*}$-algebras and their automorphism groups. London Mathematical Society Monographs, 14. Academic Press, Inc. [Harcourt Brace Jovanovich, Publishers], London-New York, 1979.

[P-GK99] Pedersen, G.K.: Pullback and pushout constructions in C*-algebra theory, J. Funct. Anal. 167 (1999) 243-344.

[R-MA90] Rieffel M.A., Noncommutative tori-a case study of noncommutative differentiable manifolds. Geometric and topological invariants of elliptic operators (Brunswick, ME, 1988), 191-211, Contemp. Math., 105, Amer. Math. Soc., Providence, RI, 1990 .

[R-MA93] Rieffel M.A., $K$-groups of $C^{*}$-algebras deformed by actions of $R^{d}$. J. Funct. Anal. 116 (1993) 199-214.

[T-M79] Takesaki M., Theory of operator algebras I, Springer-Verlag, New York Heidelberg Berlin, 1979.

[W-NE93] Wegge-Olsen N.E., $K$-theory and $C^{*}$-algebras. A friendly approach. Oxford Science Publications. The Clarendon Press, Oxford University Press, New York, 1993.

[W-SL87] Woronowicz S.L., Twisted SU(2) group. An example of a noncommutative differential calculus. Publ. Res. Inst. Math. Sci. 23 (1987) 117-181. 